\title{
Routine testing before cataract surgery did not reduce medical adverse events
}

Schein OD, Katz J, Bass EB, et al, for the Study of Medical Testing for Cataract Surgery. The value of routine preoperative medical testing before cataract surgery. N Engl J Med 2000 Jan 20;342:168-75.

QUESTION: In patients having cataract surgery, does routine pre-operative medical testing reduce medical adverse events?

Source of funding: US Agency for Health Care Policy and Research.

For correspondence: Dr O Schein, 116 Wilmer Building, Johns Hopkins Hospital, 600

\section{Design}

Randomised (allocation concealed*), blinded (outcome assessors), ${ }^{*}$ controlled trial with 1 week follow up.

\section{Setting}

9 clinical centres in the US and Canada.

\section{Patients}

18189 patients (mean age 74 y, $61 \%$ women) who were scheduled to have 19557 cataract operations between 1995 and 1997. Exclusion criteria were age $<50$ years, general anaesthesia, myocardial infarction within the previous 3 months, pre-operative medical testing in the previous 28 days, or inability to speak English or Spanish. 19250 cataract operations were done, and 19217 operations (98\% of enrolled operations) had 1 week of follow up.

\section{Intervention}

Each patient received a pre-operative medical assessment. Each patient with a scheduled cataract operation in a single eye was allocated to routine pre-operative testing ( $\mathrm{n}=9775$ operations) or no testing $(\mathrm{n}=9782$ operations). Routine testing included a 12 lead electrocardiogram; a complete blood count; and measures of serum electrolytes, urea nitrogen, creatinine, and glucose. Tests could be ordered in the no testing group only if a patient had a new or worsening medical problem that would require testing even if surgery were not planned.

\section{Main outcome measures}

Adverse events during and after surgery.

\section{Main results}

Analysis was by intention to treat. Events were counted on a per operation basis. The groups did not differ for number of adverse events (including death and subsequent hospital admissions) overall: 301 events occurred in both groups (table). On the day of surgery, 190 events occurred in the routine testing group, and 185 occurred in the no testing group (table). 116 adverse postoperative events occurred in the routine testing group, and 121 occurred in the no testing group (table). The occurrence of different types of adverse events was similar in the 2 groups, except for that of bronchospasm, which occurred 8 times in the no testing group and 2 times in the routine testing group.

\section{Conclusion}

In patients having cataract surgery, routine preoperative medical testing was no more effective than no testing in reducing medical adverse events.

*See glossary.

North Wolfe Street,

Baltimore, $M D$

21287-9019, USA.

Fax +1410614

9651 .
Adverse events associated with routine testing vs no testing before cataract surgery†

\begin{tabular}{llll} 
& \multicolumn{2}{l}{ Number of events/1000 patients } & Relative risk \\
\cline { 2 - 4 } Adverse events & Routine testing & No testing & (95\% Cl) \\
Overall & 31.3 & 31.3 & $1.0(0.9$ to 1.2$)$ \\
\hline Intraoperative & 19.7 & 19.2 & $0.97(0.8$ to 1.2$)$ \\
\hline Postoperative at 1 week & 12.1 & 12.6 & $1.04(0.8$ to 1.3$)$ \\
\hline
\end{tabular}

\section{COMMENTARY}

This well designed study by Schein $e t$ al suggests that routine laboratory evaluation is not warranted for a low risk procedure, such as cataract surgery. The study population was selected because patients presenting for this elective procedure usually do not have serious medical comorbid conditions. This fact is borne out by the high proportion (99\%) of patients who had an American Society of Anesthesiologists (ASA) classification of $\leqslant$ III. These are indeed the patients for whom we would like to confirm that we are not missing important outcomes by omitting these tests.

A more complex issue is the link between pre-operative evaluation and outcomes. In the no routine testing group in this study, patients having no tests had a lower rate of events than those having some tests (29.1/1000 operations $v 52.6 / 1000$ operations). One wonders what prompted healthcare providers to order the tests and whether acting on the results made any difference to patient outcomes. Overall, although higher ASA class and poorer health status predicted more adverse events, this stratification did not reveal differences in events between the routine testing and no testing groups.

Minor questions remain about the level of training of the screening physicians (ie, are specialists better able to predict events?) and whether the short follow up of 1 week excluded relevant events.

This study supports results from previous studies that have shown that routine preoperative laboratory tests add little to the prevention of surgical complications. ${ }^{1-3}$ Assessing the value of laboratory tests directed by an appropriate history and physical examination for patients having surgical procedures remains a research priority.

Neil Gibson, MSc, MD University of Alberta Edmonton, Alberta, Canada

1 Velanovich V. Preoperative laboratory screening based on age, gender, and concomitant medical diseases. Surgery 1994;115:56-61.

2 Wattsman TA, Davies RS. The utility of preoperative laboratory testing in general surgery patients for outpatient procedures. Am Surg 1997;63:81-90.

3 Velanovich V. The value of routine preoperative laboratory testing in predicting postoperative complications: a multivariate analysis. Surgery 1991;109:236-43. 